\title{
Lingots et déchets de fonderie dans les dépôts de l'horizon de l'épée à pointe en langue de carpe (Bronze final IIIb) : proposition de typologie
}

\author{
Cécile Le Carlier de Veslud, Laura Edme et Muriel Fily
}

Résumé : Les lingots de cuivre et les déchets de fonderie sont des objets omniprésents dans les dépôts métalliques terrestres de l'horizon de l'épée à pointe en langue de carpe du Bronze final IIIb (Bronze final atlantique 3), en particulier dans les régions proches de la façade atlantique. Et pourtant, ils ont été systématiquement sous-étudiés et même sousréférencés. Après un passage en revue des différentes descriptions de ces objets depuis un siècle, ce travail propose une typologie fine basée sur leurs descriptions morphologiques.

Cette étude s'attache à décrire les lingots et les déchets de fonderie des dépôts de l'horizon de l'épée à pointe en langue de carpe de la Bretagne historique (Finistère, Morbihan, Côtes-d'Armor, Ille-et-Vilaine, Loire-Atlantique). Ainsi, sous l'ancienne dénomination "lingots plano-convexes », plusieurs types d'objets sont en réalité décrits : d'une part les déchets de fonderie en bronze que sont les coulures sur le sol, les moulages de creusets et les moulages de fond de poterie qui doivent certainement correspondre à des surplus obtenus au moment de la fonte des objets; et d'autre part les lingots plano-convexes de cuivre qui correspondent à de la matière première importée. Enfin, les masselottes en bronze ont également été considérées. Elles sont parfaitement identifiées, mais le vocabulaire utilisé est si diversifié qu'une reprise rigoureuse de la terminologie s'est avérée indispensable.

La description fine des déchets de fonderie est particulièrement pertinente lorsque l'on s'attache à reconstituer les techniques métallurgiques des bronziers de l'époque, notamment la taille et la nature des creusets ou la reconstitution des moules. La description des lingots de cuivre est quant à elle très importante pour tenter de restituer des courants d'échange à travers l'Europe. En effet, les techniques métallurgiques peuvent présenter des spécificités d'une zone de production à une autre et la morphologie des lingots peut subtilement varier. Ainsi, la comparaison des aspects macroscopiques des lingots de cuivre trouvés dans les zones de production et dans celles de consommation, peut apporter des éléments de discussion dans ces problématiques d'échanges.

Mots clés : Métallurgie, déchets de fonderie, lingot plano-convexe, dépôt, bronze, cuivre, Bronze final IIIb atlantique, description morphologique.

Abstract: Copper ingots and foundry waste are very common in metallic dry land hoards of the carp's tongue sword horizon of the Late Bronze Age IIIb, especially in the Atlantic area (fig. 1). However, they have been systematically under-studied and even under-referenced. After an analysis of the various descriptions over time, this study proposes an accurate typology based on morphological descriptions. The main purpose of this work is to identify different types of copper ingots in order to determine if one or several copper origins could have been used to supply bronze artisans' workshops at the same time in Western Europe and most particularly in the west of France.

In 2009, the number of Late Bronze IIIb hoards known in the west of France was about sixty nine (Boulud \& Fily, 2009). Following this inventory, it can be noted that almost all these hoards contained foundry waste or/and ingots. This configuration is specific to western France, because in the rest of the country hoards are few, for example in the south of France (Guilaine, 1972; Gomez de Soto \& Milcent, 2000), or they did not contain foundry waste, for example in the north of France (Blanchet, 1984), or only very little, as in Burgundy (Mordant, 2001) or in the Alps (Fischer, 2012). Previous publications clearly show that for several decades these objects were neglected, and that today it is still difficult to have precise information regarding their descriptions.

The aim of this paper is to propose a new typology with appropriate terminology, so it could be used for all future studies to describe these objects with a single identification process. This work describes ingots and foundry waste from carp's tongue sword horizon hoards in historical Brittany (Finistère, Morbihan, Côtes-d'Armor, Ille-et-Vilaine, Loire-Atlantique). Thus, several types of objects are actually described under the old name of "plano-convex ingots": on the one hand, bronze foundry waste such as ground flows, crucible castings, and casts of the bottom of ceramic containers which must certainly be an overflow created during the casting of objects; and on the other hand the plano- 
convex copper ingots which correspond to the imported raw material. Lastly, bronze "masselottes" have also been considered in the study. They are clearly identified, but the vocabulary used is so diverse that a rigorous terminology is now indispensable.

Ground flows have an overall plano-convex profile. The form of these bronze flows depends on the geometry of the cavity in which the metal is flowing. The upper surface is generally smooth but sometimes wrinkled, the lower slightly irregular and moulds the roughness of the ground. There is no porosity (fig. 2). Crucible castings also have a planoconvex profile. The upper surface is smooth as is the lower, which moulds the inner wall of the crucible. These bronze objects also lack porosity (fig.3). Almost identical casts have been found in Devon (Needham 1980). Casts of the bottom of ceramic containers have an overall plano-convex profile. The upper surface is slightly rough or bumpy. The lower surface is smooth and corresponds to the imprint of the bottom and part of the sides of the ceramic object. These bronze objects have no porosity (fig. 4). 'Masselottes' correspond to the excess metal remaining in the funnel above objects cast in a mould. They are formed by an upper reservoir corresponding to the funnel itself, and by one or several pouring streams (fig. 5).

The plano-convex copper ingots generally have a circular or slightly oval shape with an average diameter of twenty centimetres (fig. 6). Porosity can be low but is usually very high. A large number of these ingots are fragmented and some are partially melted (fig. 7). Unlike the previous objects, ingots are not produced in local bronze artisans' workshops, but are imported from producing areas. Western France has insufficient resources for the manufacture of such a large number of objects. It is very important to describe the ingots because some differences could appear. For example some smaller ingots, maybe from another production area, could exist (fig. 8). It is nevertheless certain that other forms of ingots exist for the same period in France, as shown by the example of the biscuit-shaped ingots from the hoard of Saint-Jean-aux-Bois, Oise. European comparisons show that plano-convex ingots present in large numbers in the west of France have a morphology very strongly resembling that found in England (Roberts \& Veysey, 2011). Plano-convex ingots found in Spain are smaller (Gomez Ramos, 1993; Montero-Ruiz et al., 2011) while ingot axes are known in the east of the Iberian Peninsula (Renzi, 2010).

In conclusion, an accurate description of foundry waste is extremely pertinent for our knowledge of the ancient artisans' metallurgical technology, especially concerning the size and form of crucibles or the reconstitution of moulds. The description of copper ingots is very important for trying to identify trade routes through Europe. Indeed, metallurgical techniques can present specificities from one production area to another and ingots could be morphologically different, even if only slightly. The comparison of macroscopic aspects of copper ingots found in production areas with those of consumption areas can contribute to discussion about trade and exchange questions.

Keywords: Metallurgy, foundry waste, plano-convex ingot, hoard, bronze, copper, Atlantic Late Bronze Age, morphological description.

$\mathrm{L}$

A REPRISE du programme d'analyses chimiques des objets à base cuivre a conduit le laboratoire de paléométallurgie de l'UMR 6566 « CReAAH » à l'université Rennes 1 à s'intéresser à la totalité des objets présents dans les dépôts métalliques terrestres de l'âge du Bronze. Les lingots de cuivre et les déchets de fonderie en font partie intégrante. Ces lingots, qui sont issus directement du stade de réduction, c'est-à-dire de l'étape de transformation du minerai en métal ne doivent pas être négligés. Ils portent en eux la signature chimique du métal des zones de production. Nous avons donc tenté de les recenser afin de pouvoir les analyser. Lors de cet inventaire, l'imprécision des données et le manque de considération vis-à-vis de ce type d'artefacts sont apparus de manière flagrante. Louis Marsille en son temps faisait déjà remarquer que les " lingots " avaient été négligés (Marsille, 1911). Hormis pour les dépôts ayant contenu uniquement des « lingots », comme celui Kerbromeur à Tourc'h, Finistère (Villiers du Terrage, 1896), les publications de l'époque n'ont que très partiellement abordé le sujet. Les travaux les plus récents se sont concentrés sur leur composition chimique, via la réalisation d'analyses afin de permettre un premier classement (Briard et al., 1964-1965). Cependant, la description fine de ces objets a été la plupart du temps négligée.
Nous avons choisi dans un premier temps de concentrer l'étude sur l'horizon de l'épée à pointe en langue de carpe du Bronze final IIIb (Bronze final atlantique 3; abrégé en $\mathrm{BFa} 3$, selon la nouvelle terminologie proposée par P.-Y. Milcent, 2012). Toutefois, au vu du nombre important de ces dépôts (fig. 1) seuls les cinq départements de la Bretagne historique ont été pris en compte (Finistère, Morbihan, Côtes-d'Armor, Ille-et-Vilaine, Loire-Atlantique). Plus d'une centaine de dépôts ont ainsi été référencés. L'approche dans ce travail a été double : bibliographique d'une part, pour dénombrer les dépôts contenant des « lingots » et déchets de fonderie, et typologique d'autre part, par l'étude et la sélection de quelques ensembles.

\section{L'HORIZON DE L'ÉPÉE À POINTE EN LANGUE DE CARPE DANS L'OUEST DE LA FRANCE}

\footnotetext{
T horizon métallique de l'épée à pointe en langue de carpe présente des caractéristiques communes bien spécifiques. On y remarque une diversité fonctionnelle importante des objets avec la présence de marqueurs tels que l'épée à pointe en langue de carpe, les poignards à
} 


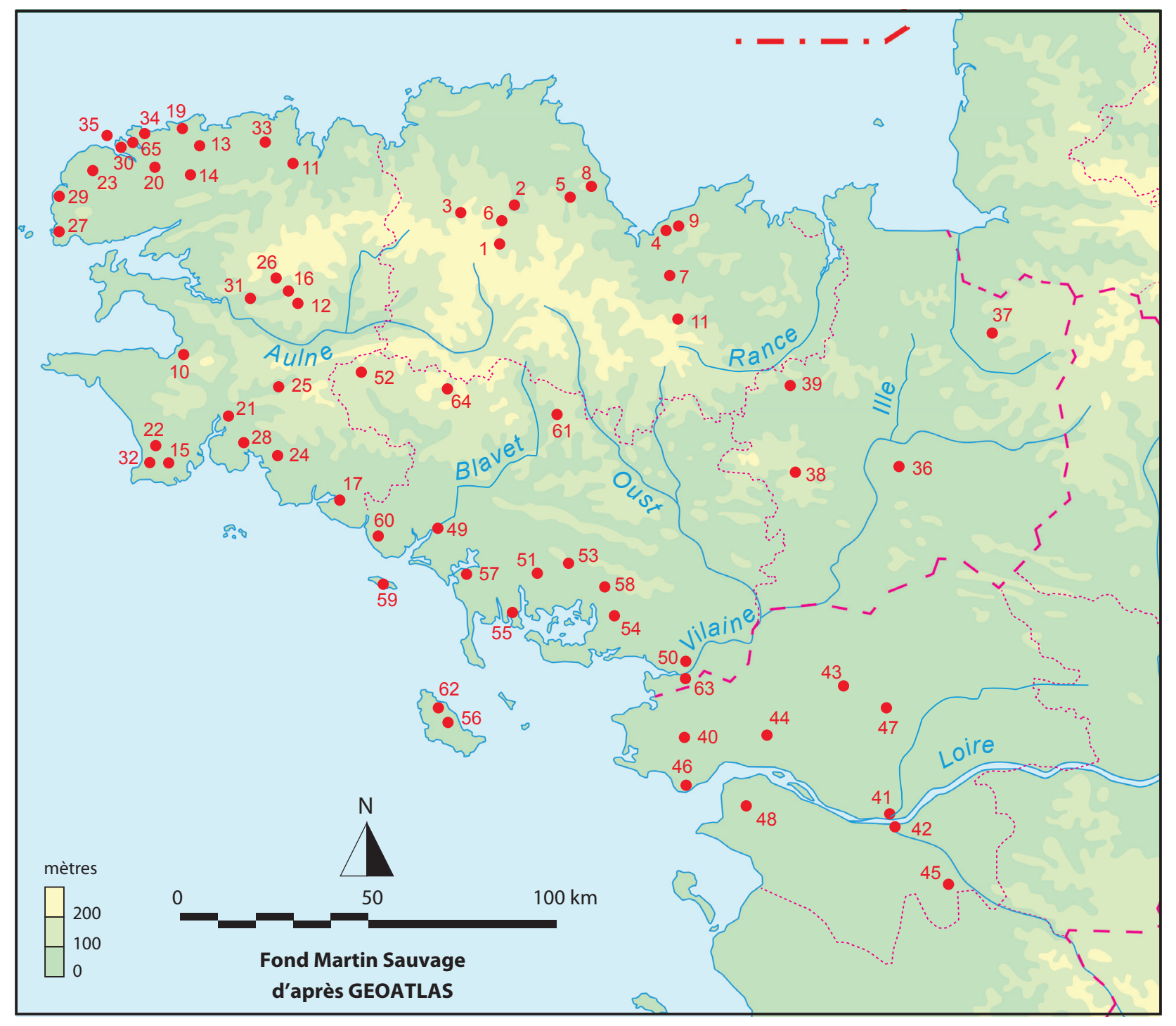

Fig. 1 - Carte de localisation des dépôts contenant des « lingots » ou des déchets de fonderie en Bretagne et en Loire-Atlantique. 1 : Maël-Pestivien (22), Guern-an-Floch; 2 : Plouisy (22), Mesguen; 3 : Plouarc'h (22), Min-ar-Groac'h; 4 : Planguenoual (22), VilleEon; 5 : Bringolo (22), Kervisis; 6 : Guingamp (22), Bourbiac; 7: Bréhand (22), Le Pont-Pierre (carrière); 8 : Étables (22), Le Petit Cahurel; 9 : Planguenoual (22), La Crapon; 10 : Kerlaz ou Plonévez-Porzay (29); 11 : Taulé (29), Kergariou; 12 : Landeleau (29), Penfoul; 13 : Goulven (29), le dolmen de Cosquer; 14 : Ploudaniel (29), Keranou; 15 : Plobannalec (29), Par-un-Puns et Lesconil; 16 : Plonevez-du-Faou (29), Kermaria; 17 : Moëlan-sur-Mer (29), Mescleo et Vern; 18: Plénée-Jugon (29), la ville Génée; 19 : Kerlouan (29); 20 : Le Folgoet (29), Pen-ar-Prat; 21 : Gouesnach (29), Menez Tosta, Lanhuron, Keragaradec 1, Kergaradec 2, Kergaradec 3; 22 : Ploneour-Lanvern(29), Keroberon; 23: Saint Pabu (29), Kerc'hleus; 24 : Beuzec-Conq (29), Bois de Lesnevar; 25 : Tourc'h (29), Kerbromeur; 26 : Loqueffret, Keryeven (29); 27 : Le Conquet (29), Kervildré; 28 : Fouesnant (29), Le Douric; 29 : LampaulPlouarzel (29); 30 : Lannilis (29), La Motte; 31 : Pleyben (29), La Boixière; 32 : Plomeur (29), Kerbulic; 33 : Plouenan (29), Le Bourg; 34 : Ploudalmézeau (29); 35 : Landeda (29), île Guennoc; 36 : Janzé (35), La Métairie-Neuve; 37 : Saint-Marc-le-Blanc (35), Le Plessis ; 38 : Plélan-Le-Grand (35); 39 : Saint-Méen-Le-Grand (35); 40 : Besné (35), Le Gros Chêne; 41 : Nantes (44) Jardin des Plantes; 42 : Nantes (44), Prairie de Mauves; 43 : Plessé (44); 44 : Campbon (44), La Gergaudais; 45 : Clisson (44); 46 : Pornichet (44), Pointe de Congrigoux Plage Sainte-Marguerite; 47 : Puceul (44), Île aux Lievres ou Chevallerais; 48 : Saint-Père-en-Retz (44), La Tiedenaie; 49 : Hennebont (56), Kerorch; 50 : La Roche-Bernard (56), Nivillac; 51 : Plougoumelen (56); 52 : Roudouallec (56), Kerhon; 53 : Bignan (56), Keran; 54 : Questembert (56), Parc-aux-Bœufs; 55 : Locmariaquer (56), Pont-er-Vil; 56 : Bangor (56), Calastrène (Belle-Île); 57 : Belz (56), île Nihen; 58 : Elven (56), Le Bocolo; 59 : Ile de Groix (56), Men-Strang-Roth; 60 : Guidel (56), Kergal et Kerhar; 61 : Noyal-Pontivy (56), Le Couedic; 62 : Le Palais (56), Bordustrat (Belle-Île); 63 : La Roche-Bernard (56); 64 : Langoelan (56), Botcazo; 65 : Plouguerneau (29), Kergadavarn (carte L. Quesnel).

Fig. 1 - Location map of hoards in Brittany containing copper ingots or bronze foundry waste. 
languette, pointes de lance, haches à ailerons, haches à douille essentiellement du type du Plainseau, racloirs et autres outils, bracelets, éléments de harnachement et pièces de char, débris de vaisselle, lingots plano-convexes et déchets de fonderie. Les objets de cet horizon ont subi une grande fragmentation, et ils ne sont que très rarement représentés dans leur globalité (Briard, 1965; Boulud et Fily, 2009).

Le taux très important de fracturation et le nombre imposant d'objets liés à la métallurgie, ont conduit, dès les premières découvertes étudiées au XIX ${ }^{\mathrm{e}}$ siècle et longtemps ensuite, une grande partie des chercheurs à les qualifier de dépôts de fondeurs : il s'agirait de cachettes réalisées en vue du recyclage de métaux, et qui auraient été abandonnées à cause d'une insécurité générale. Mais comment expliquer alors que plusieurs milliers de dépôts aient été retrouvés à travers l'Europe? Cette hypothèse de dépôts de fondeurs est aujourd'hui considérée comme peu probable, et il semblerait que ces ensembles soient plutôt liés à un processus cultuel ou tout du moins social.

Le travail présenté ici a été rendu possible grâce à l'inventaire récent et rigoureux entrepris par S. Boulud et l'une d'entre nous (Boulud et Fily, 2009). Ainsi, au moment de cette publication, le nombre total de dépôts s'élevait à soixante-neuf répartis très inégalement entre les cinq départements considérés (huit dans les Côtes-d'Armor, trente-trois dans le Finistère, cinq en Ille-et-Vilaine, dix en Loire-Atlantique et treize dans le Morbihan). À la suite de ce travail, il a été possible de déterminer les dépôts qui contenaient des déchets de fonderie et ceux qui n'en contenaient pas. Cependant, la composition précise de l'ensemble de ces dépôts n'a pas toujours pu être établie faute d'inventaires précis ou complets lors de leur découverte, car ceux-ci ont souvent été amputés d'une partie de leurs pièces, lors de la mise de côté par les découvreurs ou les collectionneurs des plus beaux objets, ou lors de la refonte quasi-immédiate de pièces jugées sans intérêt, dont une très grande partie des déchets de fonderie et des lingots plano-convexes.

Si certains de ces dépôts ne contiennent que quelques objets (celui de Louvigné-du-Désert en Ille-et-Vilaine n'en contenait que huit), d'autres se composent de plus d'une centaine comme le dépôt de la Prairie de Mauves à Nantes avec cent cinquante-cinq objets (Briard, 1965; Boulud et Fily, 2009). Cependant, l'étude a montré que plus de quatre-vingts pour cent des dépôts contenaient des déchets de fonderie ou des lingots, et si l'on suppose que beaucoup ont été omis des inventaires, on peut considérer alors que presque la totalité de ces dépôts pourraient contenir ce type de matériel. Il faut néanmoins remarquer que ce ne sont pas les dépôts contenant le plus grand nombre d'objets qui possèdent nécessairement la plus grande part de résidus de fonderie et de lingots, d'après l'exemple du dépôt du Jardin des Plantes à Nantes (Briard, 1971). Ce dépôt est composé de cent cinquante-cinq objets dont seulement dix-neuf objets liés à la métallurgie, décompte effectué après visite au musée Dobrée.

\section{LES DÉPÔTS TERRESTRES DU BRONZE FINAL IIIB EN FRANCE ET LE PARTICULARISME DU NORD-OUEST}

$\mathrm{D}$ ans l'ensemble du territoire français, des objets en métal ont été retrouvés, que ce soit de manière isolée ou qu'ils soient regroupés en dépôts. Le but de cette partie n'est pas de faire une liste exhaustive de ceux-ci, ni une description précise de leur composition, mais de mettre en évidence certaines caractéristiques apparues lors de la recherche bibliographique à partir de travaux de synthèse. Il faut noter que les lingots et les déchets de fonderie n'ont jamais fait l'objet d'études fines, il est donc difficile de se faire une idée précise de la présence et de la nature de ces objets dans les dépôts à partir des synthèses régionales déjà réalisées (voir infra). Sous la dénomination « lingots » se cachent aussi bien des pièces en cuivre qu'en bronze. Souvent, le simple fait qu'ils ne ressemblent pas à des objets manufacturés suffit à les faire entrer dans la catégorie « lingot».

Dans les Alpes, les dépôts retrouvés en contexte palafittique ne contiennent que très peu d'éléments liés à la métallurgie, essentiellement des déchets de fonderie. Un seul lingot plano-convexe entier et deux fragments ont été retrouvés (Fischer, 2012). Dans les Alpes-Maritimes, il existe des dépôts, mais ceux-ci ne semblent composés que d'objets manufacturés (Mercurin, 2011). Il en est presque de même dans les Flandres où les dépôts sont très peu nombreux, sans mention d'objets liés à la métallurgie (Bourgeois et Cherreté, 2005). En Picardie et dans le Nord de la France, il y a de nombreux dépôts, surtout composés d'objets variés et de peu de déchets de fonderie. Les lingots de cuivre, s'ils existent, ne sont pas mentionnés (Blanchet, 1984). En Lorraine et Sarre, où les dépôts sont moins nombreux que dans l'Ouest, quelques éléments de fonderie et des lingots de bronze sont mentionnés, mais pas de lingots de cuivre (Véber, 1996). En Bourgogne, une majorité de dépôts ne présenteraient pas de déchets de fonderie ni de lingots (Gauthier, 2003). Les quelques rares dépôts à en posséder ne sont cependant pas particulièrement riches puisqu'on ne cite bien souvent qu'un seul lingot (Mordant, 1996). Cependant, ce lingot est de type plano-convexe et ses dimensions correspondent tout à fait à ceux rencontrés dans les dépôts du Nord-Ouest (Mordant, 2001). Dans le Jura et les plaines de la Saône, les éléments liés à la fonderie sont extrêmement rares et ceux-ci semblent ne se retrouver qu'en contexte d'habitat (Gauthier, 2009). Dans la région de Paris, les dépôts possèderaient fréquemment des fragments de lingots, sans plus de précision (Mohen, 1977). Dans la région Centre, seul le dépôt du Petit Villatte (Cordier, 1996; Milcent, 1998) possède quatre lingots. La bibliographie relative aux dépôts est peu nombreuse dans le Sud de la France. Actuellement, ces dépôts semblent quasi-inexistants (Guilaine, 1972; Gomez de Soto et Milcent, 2000).

En Centre-Ouest, des lingots de cuivre sont mentionnés dans les dépôts du Poitou (Pautreau, 1979 et 1984). 
On notera par exemple la grande quantité de fragments dans le dépôt de Challans (Verney, 1990) et d'autres dans le dépôt de Vénat (Coffyn, 1971; Coffyn et al., 1981).

Cependant, les dépôts contenant de grandes quantités de lingots de cuivre ou composés uniquement de lingots semblent se concentrer plus particulièrement sur la façade sud du Massif armoricain, du Sud du Finistère jusqu'en Vendée (Briard, 1984). Les découvertes les plus récentes semblent confirmer ce fait, comme par exemple un des dépôts de Kergaradec à Gouesnac'h (Finistère), composé uniquement de lingots (Fily, 2009).

Dans le quart nord-ouest de la France, la présence des déchets de fonderie et des lingots en grande quantité dans de nombreux dépôts est donc une caractéristique importante, déjà constatée précédemment pour la Bretagne.

\section{HISTORIQUE DES ÉTUDES PRÉCÉDENTES ET ÉTAT DE LA TYPOLOGIE EXISTANTE}

$\mathrm{I}_{\mathrm{c}}^{\mathrm{s}}$ a été possible de dénombrer soixante-neuf dépôts contenant des « lingots » ou des déchets de fonderie. La majorité, soit quarante et un, a été mise au jour au $\mathrm{XIX}^{\mathrm{e}}$ siècle. Le plus ancien dépôt recensé est celui de Calastrène à Bangor sur l'île de Belle-Île (Closmadeuc, 1863). Au XX ${ }^{\mathrm{e}}$ siècle, on en comptabilise vingt-sept et au $\mathrm{XXI}^{\mathrm{e}}$ siècle, quatre (dont les trois dépôts de Kergaradec à Gouesnac'h dans le Finistère (Fily, 2009).

Dans les publications du XIX $\mathrm{X}^{\mathrm{e}}$ siècle et de la première moitié du $\mathrm{XX}^{\mathrm{e}}$ siècle, de très nombreux termes sont utilisés pour désigner les « lingots », les « culots » et les déchets de fonderie. La dénomination des objets change d'une publication à une autre sans aucune raison. Ainsi, il arrive que le mot « lingot » soit remplacé par « culot» comme dans le cas du dépôt de Lanhuron à Gouesnac'h. R.-F. Le Men (1876) parle de « lingots », puis G. de Mortillet (1894) les nomme " culots », enfin P. du Châtellier (1899) reprend la dénomination de R.-F. Le Men. Dans la majorité des publications, ces objets ne sont que peu décrits. Il arrive que le dépôt soit entièrement représenté à l'exception de ce type de mobilier, par exemple pour le dépôt de Lampaul-Plouarzel, Finistère (Lagarde, 1863), ou que seuls quelques objets soient dessinés, par exemple pour le dépôt du Jardin des Plantes à Nantes (Parenteau, 1868) dans lequel trois masselottes (là nommées «bouchon de coulée ») sur neuf sont représentées. Les mesures, taille et poids, ne sont pas systématiques, mais restent courantes. Quant à la nature du métal, elle n'est pas toujours précisée. Il est parfois fait mention d'objets en cuivre lorsque de rares analyses chimiques ont pu être réalisées en laboratoire.

La détermination de la nature de ces objets a pu également se heurter à certains courants de pensée développés par d'éminents chercheurs, qui ont fait force de loi et qui ont pu nuire à la discussion. Ainsi, E. Villers du Terrage (1896), après la découverte du dépôt de lingots de Kerbromeur à Tourc'h, (Finistère) commande une analyse chimique et détermine ainsi que ce dépôt n'est constitué que de lingots de cuivre. Tous les objets présentant exactement les mêmes caractéristiques, il émet l'hypothèse d'une possible régression de la société à la fin de l'âge du Bronze avec un retour à la métallurgie du cuivre, et n'évoque pas la possibilité de matière première. P. du Châtellier (1899), quant à lui, ne croit pas en la présence de lingots en cuivre dans le dépôt de Lampaul-Plouarzel (Finistère). Il conteste les analyses de D. Lagarde (1863) : pour lui, les objets seraient en cuivre, l'étain ayant disparu suite aux nombreuses refontes.

C'est avec la seconde moitié du $\mathrm{xx}^{\mathrm{e}}$ siècle que les choses évoluent légèrement, puisqu'il peut y avoir encore des imprécisions concernant le dénombrement des objets liés à la métallurgie, et cela pour un même auteur! Ainsi, L. Marsille n'évoque aucun objet issu de la métallurgie en 1913 dans le dépôt de Parc-aux-Bœufs à Questembert (Morbihan), alors qu'en 1921, il dénombre trois lingots. Les objets de ce type peuvent être correctement répertoriés ou être juste mentionnés. Avec l'avancée de la recherche, les termes les désignant évoluent également mais en se diversifiant. Les termes « lingots » ou « fragments de lingots » font leur apparition. Les auteurs s'intéressent déjà plus à ces objets, mais utilisent alors un panel important de termes pour les désigner, contribuant ainsi à rendre la compréhension difficile. Cependant, certains termes disparaissent définitivement. Ainsi, les « masses de cuivre rosette » (Closmadeuc, 1863) sont devenus des lingots plano-convexes de cuivre (Briard, 1965 et 1991). Les prises de mesure sont plus courantes, mais elles ne sont pas généralisées. De plus, ce sont surtout les poids qui sont relevés et non les dimensions. Des dépôts découverts au XIX ${ }^{\mathrm{e}}$ siècle sont repris, mais de fait, leurs inventaires changent : il n'y a plus le même nombre d'objets et certains objets disparaissent. Les dessins restent souvent approximatifs si bien que certaines fois, les formes véritables sont à peine révélées.

Les photographies apparaissent dans la seconde moitié du $\mathrm{XX}^{\mathrm{e}}$ siècle mais sont surtout dévolues aux objets manufacturés. Les analyses chimiques sont plus courantes. P.-R. Giot, J. Briard, et J.-R. Bourhis ont entrepris toute une série d'analyses sur divers objets issus de nombreux dépôts (Briard et al., 1964-1965). Elles ont permis de définir plus précisément certains objets : les supposés lingots de bronze du dépôt de Lanhuron à Gouesnac'h (Le Men, 1876) sont ainsi identifiés comme des lingots plano-convexes de cuivre.

Désormais, la totalité des lingots et déchets de fonderie sont pris en compte dans les inventaires avec dessins et couverture photographique. Les analyses chimiques deviennent systématiques autant pour les objets manufacturés que pour les objets liés à la métallurgie.

Notre synthèse montrera que sous la même appellation peuvent se cacher des réalités différentes, à savoir des objets de natures diverses. Ainsi, le terme « culot » est souvent utilisé, au même titre que le mot « lingot », qu'il soit en bronze ou en cuivre. La confusion dans l'attribution typologique de ces objets reste encore d'actualité. 


\section{NOUVELLE TYPOLOGIE DES LINGOTS ET DÉCHETS DE FONDERIE EN RELATION AVEC LEUR MODE DE PRODUCTION}

$\mathrm{L}$ a confusion entre les différents types vient du fait qu'un certain nombre des objets liés à la métallurgie sont de profil globalement plano-convexe. Sans un examen morphologique détaillé, sans analyse chimique, et surtout, sans connaissance des gestes des artisans bronziers, qui permettent de porter un regard neuf sur ces objets, une part importante de ces appellations approximatives ou erronées peut être compréhensible.

Quatre grandes catégories d'objets ont pu être déterminées à l'issue de ce travail, qui étaient le plus souvent réunis sous les appellations de lingots ou culots : les écoulements sur un sol, les moulages de creuset, les moulages de fonds de céramique et les lingots planoconvexes. Un dernier type d'objets a toujours été nettement différencié des autres. Ce sont les masselottes qui correspondent aux surplus produits au moment des coulées dans les moules. Cependant, au cours des décennies précédentes, tout un panel de termes a été utilisé (selon le cas, culot de coulée, masselotte de bronze, bouchon de coulée, cône de coulée, souche à clous ou arbres de coulées), si bien qu'il est nécessaire de fixer le vocabulaire descriptif.

\section{Les écoulements sur un sol}

Ces objets se sont formés par écoulement d'un liquide métallique en fusion dans des irrégularités en creux du sol. De profil, la forme de tels objets est globalement plano-convexe, mais pas toujours car certaines coulures peuvent se présenter très simplement sous forme de langue tandis que d'autres correspondent à de petites gouttes ou nodules plus ou moins informes (fig. 2). La surface supérieure est globalement plane. Son aspect est relativement lisse mais peut être également parfois ridée. Il peut y avoir cependant des irrégularités en relief un peu plus marquées en forme de petits granules (2-3 mm de côté). La surface inférieure, généralement convexe, moule la cavité du sol, d'où la présence de nombreuses irrégularités. La grande particularité de ces objets est de ne posséder aucune porosité, ni en surface supérieure, ni au niveau de la surface inférieure. Vue de dessus, la morphologie est quelconque car elle est liée à la forme de la cavité dans laquelle le métal en fusion a coulé. Cependant, la morphologie peut être presque circulaire pour peu que la cavité ait des contours réguliers. Parfois, sur l'un des côtés, une racine peut être observée. Il s'agit du débouché d'une rigole d'où vient le métal en fusion. Jusqu'à maintenant, tous les objets étudiés et classés dans cette catégorie sont en bronze et non en cuivre.

Ces objets sont probablement produits au sein des ateliers de bronziers, au moment où sont moulés les objets. Ils pourraient correspondre soit à des éclaboussures sur le sol pour les plus petits, correspondant par exemple à une maladresse du fondeur; soit à un geste volontaire, c'est-àdire couler le surplus de métal (quand le moule est plein) dans une cavité du sol afin de le récupérer en fin d'opération pour le recycler. La recherche bibliographique effectuée a montré que ces objets peuvent être également présents dans les dépôts des autres régions françaises, et certains ont été classés en tant que lingots plano-convexes ou même en tant que moulages de creusets.

\section{Les moulages de creusets}

Un autre type d'objets, constitués uniquement par du bronze, présente également un profil globalement planoconvexe. Ce sont des masses métalliques qui se sont refroidies dans des récipients à parois relativement lisses et qui possèdent un bec verseur (fig. 3). Ils sembleraient correspondre à des creusets de bronziers. Il en existe différentes formes et tailles. La plus commune rencontrée jusque maintenant correspond à un contour quasi-circulaire, vue de dessus, de diamètre d'environ $12 \mathrm{~cm}$. Les autres formes, quasi-circulaires également, présentent une taille plus importante et une profondeur moindre, ou une taille plus petite. La particularité de ces objets est également l'absence de porosité en surface supérieure comme inférieure. La surface supérieure est globalement plane et des portions montrent un aspect particulièrement lisse. Des irrégularités sont marquées par la présence en relief de particules dont la taille varie du granule au gravier, souvent anguleux, rugueux, parfois avec une allure déchiquetée. La surface inférieure convexe est très légèrement granuleuse du fait de la nature du récipient qui ne devait pas être parfaitement lisse, ou alors du fait qu'ils ont servi plusieurs fois avec soit arrachages, soit incrustations de restes de métal d'opérations précédentes.

Actuellement, peu de creusets sont connus pour l'âge du Bronze en France, et la majorité a été trouvée au Fort
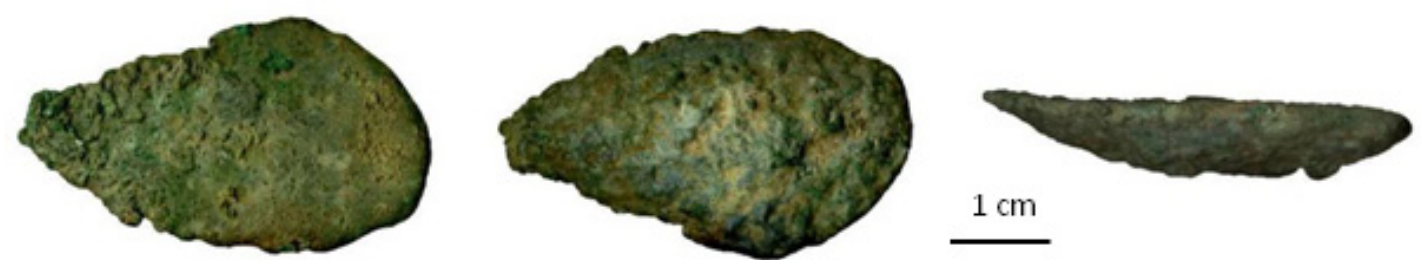

$1 \mathrm{~cm}$

Fig. 2 - Exemple d'écoulement sur un sol (dépôt de Kergaradec, Gouesnac'h, Finistère).

Fig. 2 - Example of ground flow (Kergaradec hoard, Gouesnac'h, Finistère). 

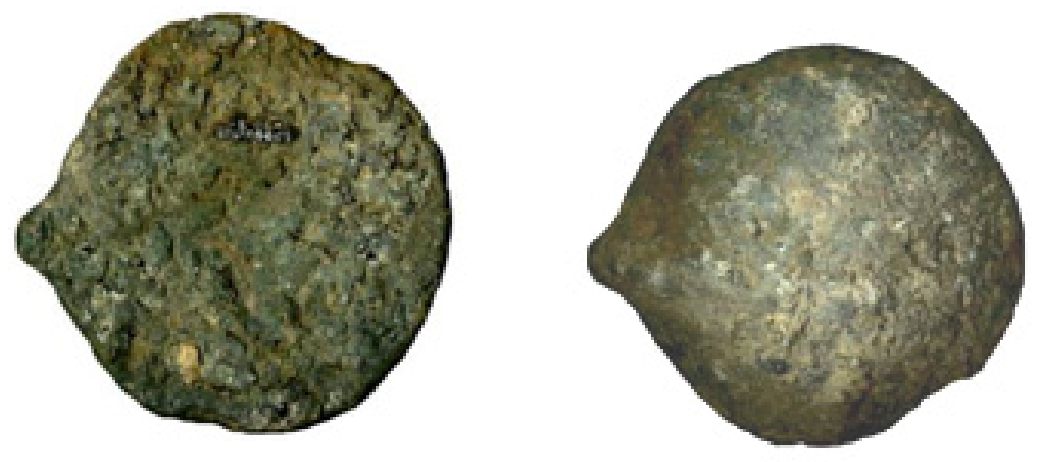

\section{$1 \mathrm{~cm}$}

Fig. 3 - Exemple de moulage de creuset (dépôt de Kergaradec, Gouesnac'h, Finistère).

Fig. 3 - Example of crucible casting (Kergaradec hoard, Gouesnac'h, Finistère).


$1 \mathrm{~cm}$

Fig. 4 - Exemple de fond de pot céramique (dépôt de Kergaradec, Gouesnac'h, Finistère)

Fig. 4 - Example of a cast of the bottom of a ceramic container (Kergaradec hoard, Gouesnac'h, Finistère).

Harrouard (Queixalos et al., 1987). Ils sont majoritairement larges et peu profonds et un seul bec verseur est attesté. Des fragments de creusets trouvés dans le Devon (Needham, 1980) présentent, une fois reconstitués, des formes beaucoup plus proches des moulages présentés ici. L'hypothèse de moulage de creusets semble bien se confirmer et ce métal pourrait correspondre au reliquat de métal fondu qui aurait été produit en surplus et qu'on aurait laissé refroidir dans son récipient. Une fois solidifié, le métal se démoule aisément, laissant le creuset intact pour une nouvelle opération.

\section{Les moulages de fonds de pots en céramique}

Un troisième type d'objets en bronze présente aussi un profil globalement plano-convexe. Il s'agit toutefois d'un profil particulier et non régulier (fig. 4) puisqu'il s'agit de pièces métalliques moulant la base des céramiques. Il n'y a pas de taille standardisée puisqu'elle correspond à la dimension du pot. Il faut compter aussi avec le niveau de remplissage dans le pot qui induit un diamètre plus ou moins large pour un même type de contenant. La surface supérieure est globalement plane. Elle peut être lisse et peu bosselée ou rugueuse et légèrement irrégulière comme s'il y avait présence de granules. La surface inférieure est particulière lisse puisqu'elle moule une céramique. Cette surface inférieure correspond au fond et à la base de la panse du pot. Il n'y a aucune porosité visible. Le profil de la surface inférieure correspond parfaitement aux profils que l'on connait pour les céramiques de cette époque. Certaines collections de céramiques de l'Ouest de la France ont été bien décrites récemment (Marcigny et al., 2005).

Il semblerait que pour le Bronze final aucune céramique scorifiée n'ait été trouvée. Par contre, pour le Bronze moyen, une céramique trouvée dans la grotte des Agris (Gomez de Soto, 1995) indique clairement que des céramiques, à usage domestique à l'origine, ont été utilisées pour y verser du métal. Pendant des années, les auteurs les ont cités comme étant des moules à lingots de bronze. Cette dernière hypothèse implique la volonté de les fabriquer en vue d'une « commercialisation », d'un échange. Ils doivent alors faire partie de la chaîne opératoire. Devant la variété de forme des moulages en bronze, qui est liée à la taille de la céramique et de son inclinaison au moment de la coulée, ces rares objets semblent plus correspondre à un geste du fondeur, délibéré certes, au cours de ses opérations en vue de récupérer le métal, pour recyclage probablement.

\section{Les masselottes}

Ces objets en bronze correspondent au surplus de métal qui se positionne dans l'entonnoir au-dessus des objets 




$1 \mathrm{~cm}$
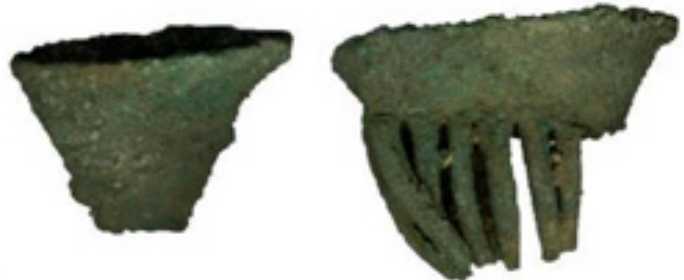

Fig. 5 - Exemples de masselottes (dépôt de Kergaradec, Gouesnac'h, Finistère).

Fig. 5 - Example of masselottes (Kergaradec hoard, Gouesnac'h, Finistère).

coulés dans le moule (fig. 5). Ils sont composés de deux parties : un réservoir supérieur créé spécialement pour absorber les retassures (fentes produites par la rétractation du métal au cours de son refroidissement), et d'un ou plusieurs canaux d'écoulement qui permettent l'alimentation en métal liquide pour le moulage des objets. Dans la terminologie technique du travail du bronze, la partie supérieure s'appelle masselotte ou entonnoir de coulée et les canaux s'appellent jets. Il n'y a pas de terme pour décrire l'assemblage de ces deux parties. On peut donc proposer que l'ensemble s'appelle masselotte, que la masse supérieure s'appelle entonnoir et que les canaux s'appellent jets. La forme de l'ensemble est particulièrement variée en fonction de la nature des objets à fabriquer et de leur nombre.
En ce qui concerne l'entonnoir, sa taille est proportionnelle à la taille des objets qui doivent être fabriqués. Il semble également que la forme soit plus ou moins liée au type d'objet fabriqué. Lorsque la masse de métal doit être importante, cette partie présente plutôt un profil en cône car c'est la forme la plus facile à réaliser (fig. 5). Lorsque la masse de métal ne doit pas être trop importante, le moule est à peine creusé et cette partie ressemble alors à une sorte de languette à partir de laquelle partent les jets de coulée (fig. 5). L'aspect de la surface supérieure est granuleux et il est possible quelquefois d'y observer de petites fissures, correspondant aux retassures. Le bord du cône est globalement lisse bien que très légèrement granuleux. Il s'agit donc de l'empreinte de la partie supérieure du moule. Pour les jets de cou- a


b


c


$1 \mathrm{~cm}$

Fig. 6 - Exemples de lingots plano-convexes de cuivre (dépôt de Kergaradec, Gouesnac'h, Finistère).

Fig. 6 - Example of copper plano-convex ingots (Kergaradec hoard, Gouesnac'h, Finistère). 
lée, la forme du canal d'écoulement est directement en rapport avec la forme de l'objet qui doit être fabriqué. Sa forme peut être circulaire ou plutôt ovalaire, ou anguleuse à base carrée ou losangique. Certains canaux sont très longs, d'autres sont quasiment inexistants. Leur extrémité correspond toujours à une cassure, qui est la trace de la séparation de la masselotte avec l'objet. Cette cassure doit se faire au raz de l'objet. Beaucoup de ces canaux possèdent des bavures marquant la zone de jonction des deux valves du moule.

Ces artefacts sont produits dans l'atelier du bronzier, au moment où l'artisan coule le métal en fusion dans le moule. Ce sont les seuls objets métallurgiques des dépôts qui ne présentent jamais de problème de détermination.

\section{Les lingots plano-convexes de cuivre}

Ils possèdent tous une porosité plus ou moins importante, mais omniprésente. C'est également ce paramètre qui permet de faire la différence entre ces lingots de cuivre et les résidus de fonderie. La porosité vient du fait que le métal en fusion a coulé sur une matière qui n'a pas totalement perdu son eau de constitution (une céramique cuite est au contraire très fortement déshydratée) et sous l'effet de la chaleur, il y a une réaction de dégazage. Des bulles se forment et remontent en traversant le métal liquide et éclatent en surface supérieure ou peuvent rester emprisonnées dans la masse lorsque le métal se fige. La porosité se présente sous différents aspects : ce peut être de tous petits trous en surface supérieure, mais également des cavités de taille centimétrique localisées en surface inférieure au niveau de la périphérie.

Vus de dessus, les lingots de cuivre étudiés dans ce travail ont une forme globalement circulaire, voire légèrement ovale avec un diamètre moyen autour d'une vingtaine de centimètre. Plusieurs types de lingots sont repérables en fonction de leur forme. Certains sont parfaitement plano-convexes (fig. 6a), d'autres sont plus aplatis, la convexité est alors à peine visible (fig. 6b). Il peut $\mathrm{y}$ avoir aussi des profils concavo-convexes (fig. 6c). Selon le type de lingot, la surface supérieure peut être parfaitement plane et lisse, ou alors plane mais crevée de nombreuses vacuoles. Elle peut être également granuleuse, percée de trous et donc présenter un aspect irrégulier. De même, la surface inférieure peut présenter une convexité régulière, presque lisse, ou alors être irrégulière et percée de nombreuses vacuoles localisées plus particulièrement sur la bordure.
Lorsque le lingot est fragmenté, la cassure présente un aspect particulier, avec une succession de prismes en relief qui vont du bas en haut de l'échantillon (fig. 7a). Ils correspondent aux grains métalliques qui ont eu une croissance orientée par le gradient de chaleur. La présence de ces prismes indiquerait que ces lingots ont été cassés à une température suffisamment faible pour que cette structure interne ne soit pas effacée. Des expérimentations récentes indiquent que le chauffage du bronze aux alentours de $500^{\circ} \mathrm{C}$ rend ce dernier extrêmement fragile (communication personnelle de A. Lehoërff, P.-Y. Milcent et J. Gomez de Soto au sujet d'une expérimentation réalisée au colloque Pezzi scelti de Rome en février 2012). Cependant, il faut encore vérifier que l'aspect des cassures présente les mêmes stigmates. Pour certains fragments de lingots, il est impossible de restituer leur forme et leur taille initiale et il est impossible de reconnaître la surface supérieure de la surface inférieure. La cause de ceci serait leur passage dans un foyer qui a pu provoquer un début de fusion. Tous les bords sont très arrondis et bosselés avec une impression de ramollissement important (fig. 7b). Il y a alors présence de grandes vacuoles centimétriques informes et arrondies au sein de l'échantillon.

La différence fondamentale de ces lingots avec les autres objets liés à la métallurgie concerne leur place dans la chaîne opératoire. Ainsi, tous les objets en bronze cités dans les paragraphes précédents correspondent à des surplus de coulée produits dans l'atelier de bronzier au moment de la fabrication des objets. Les lingots de cuivre, quant à eux, sont produits lors d'une étape précédente, lors de la transformation du minerai en métal, soit la phase de réduction. Ces lingots sont produits dans la région d'origine, qui n'est pas bretonne, et sont donc importés pour fournir la matière première aux ateliers des bronziers.

\section{Des objets de nature non encore déterminée, ou pouvant poser des problèmes de détermination}

Certains objets sont encore relativement inclassables. Ils ont été actuellement peu rencontrés. Cependant, leur présence pose question. Nous ne pouvons actuellement pas y répondre ou avec difficultés. Deux cas ont été rencontrés :

- le premier correspond à l'existence possible de lingots plano-convexes de cuivre de petite taille (fig. 8). En effet, dans les dépôts de Kergaradec à Gouesnach, et de



Fig. 7 - Cassure de lingots plano-convexes de cuivre. $7 \mathrm{a}:$ cassure à froid; $7 \mathrm{~b}$ : cassure avec ramollissement dû à la chaleur (dépôt de Kergaradec, Gouesnac'h, Finistère).

Fig. 7 - Breakage of copper plano-convex ingots. 7a: cold breakage; 7b: breakage with softening due to heat (Kergaradec hoard, Gouesnac'h, Finistère). 

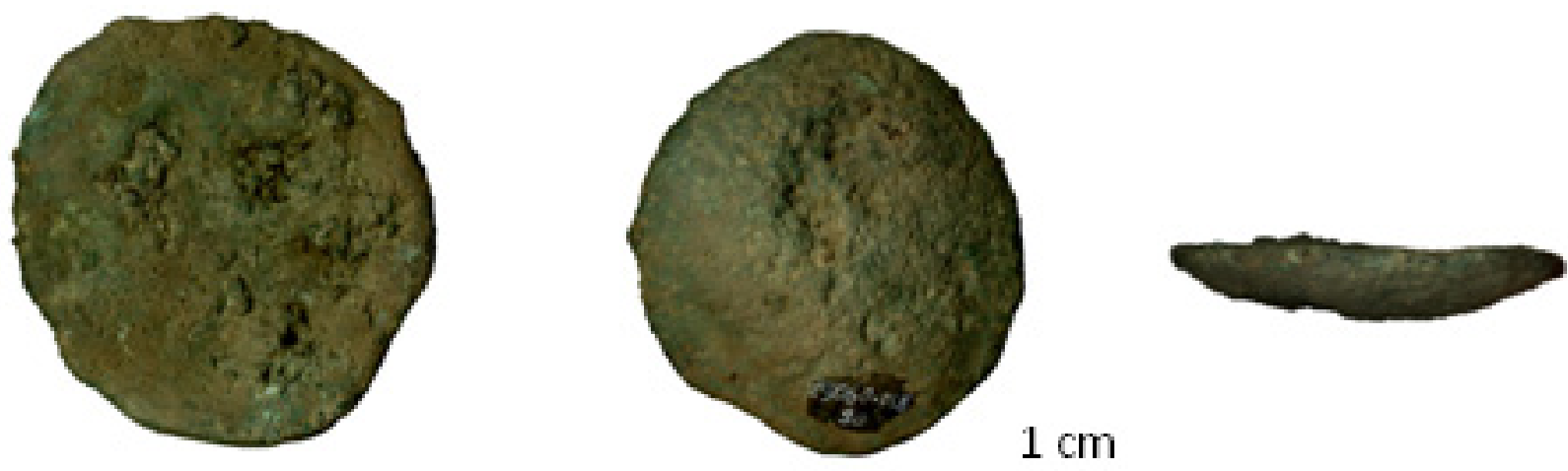

Fig. 8 - Lingot plano-convexe de cuivre de petit diamètre (dépôt de Kergaradec, Gouesnac'h, Finistère).

Fig. 8 - Small copper plano-convex ingot (Kergaradec hoard, Gouesnac'h, Finistère).

Kergadavarn à Plouguerneau ont été trouvés des éléments plano-convexes d'assez petite taille (diamètre de $10 \mathrm{~cm}$ environ) constitués de cuivre et non de bronze. À priori, ils pourraient être comparés à des coulures sur un sol car ils moulent bien des irrégularités du sol et ne présentent aucune porosité. Cependant, le manque de racine et la forme parfaitement ronde de ces deux objets sont troublants. Il est nécessaire d'acquérir de nouvelles données sur d'autres dépôts pour donner plus de consistance à cette hypothèse. Par contre, si d'autres objets de ce type sont recensés, c'est-à-dire avec une forme ronde, l'hypothèse pencherait alors plutôt en faveur de lingots planoconvexes de cuivre de petite taille issus de la réduction. De petits lingots de cuivre sont décrits en Espagne pour la même période (Gomez Ramos, 1993; Montero Ruiz et al., 2011), mais ils présentent des porosités comme les lingots plano-convexes de plus grande taille, ce qui n'est pas le cas pour le type présenté ici. Un lingot planoconvexe sans porosité de moins de dix centimètres de diamètre a été retrouvé sur le site de Corcelettes (lac de Neuchâtel). Une dizaine de lingots ont été trouvés sur le site d'Auvernier (lac Léman), mais les descriptions ne permettent pas de savoir s'il s'agit de cuivre ou de bronze (Fischer, 2012);

- le second cas correspond à l'existence possible de lingots plano-convexes de bronze. En effet, dans le dépôt de Menez Tosta, a été observé un élément en forme de galette de taille comparable aux lingots plano-convexe de cuivre (entre 15 et $20 \mathrm{~cm}$ de diamètre), sans porosité, et avec des surfaces inférieure et supérieure très lisses. Il est composé par du bronze (fig. 9). Cependant, un très léger renflement sur la face inférieure pourrait laisser penser qu'il s'agit d'un fond de creuset. Ce dernier serait alors très large et peu profond et d'une contenance très importante puisque l'objet en bronze pèse plus de trois kilos. Cet élément reste actuellement unique. Les prochaines études de mobilier sur les autres dépôts permettront de savoir s'il constitue une pièce exceptionnelle ou si la répétition est possible. Si cette détermination n'est pas exacte, il faudra admettre qu'il puisse exister un échange de métal sous forme de bronze déjà élaboré. Se poseront alors les questions suivantes : ce mélange est-il fabriqué par des ateliers de bronzier spécialisé (fabrication spécifique, ou simple moyen commode de transporter du métal de récupération avec un faible encombrement)? Est-il produit dans les zones de production de cuivre? Ou alors, l'atelier se trouve-t-il dans les zones de production d'objets? Pour alimenter la discussion, on pourra noter que de petits lingots plano-convexes en bronze ont été retrouvés en Espagne, pour la même époque, mais ces derniers sont de taille bien moindre et présentent une contour ovalaire (Gomez Ramos, 1993; Montero Ruiz et al., 2011). Il n'y a aucune ressemblance avec l'objet présenté ici, mais cela montre néanmoins que des lingots de bronze peuvent exister.
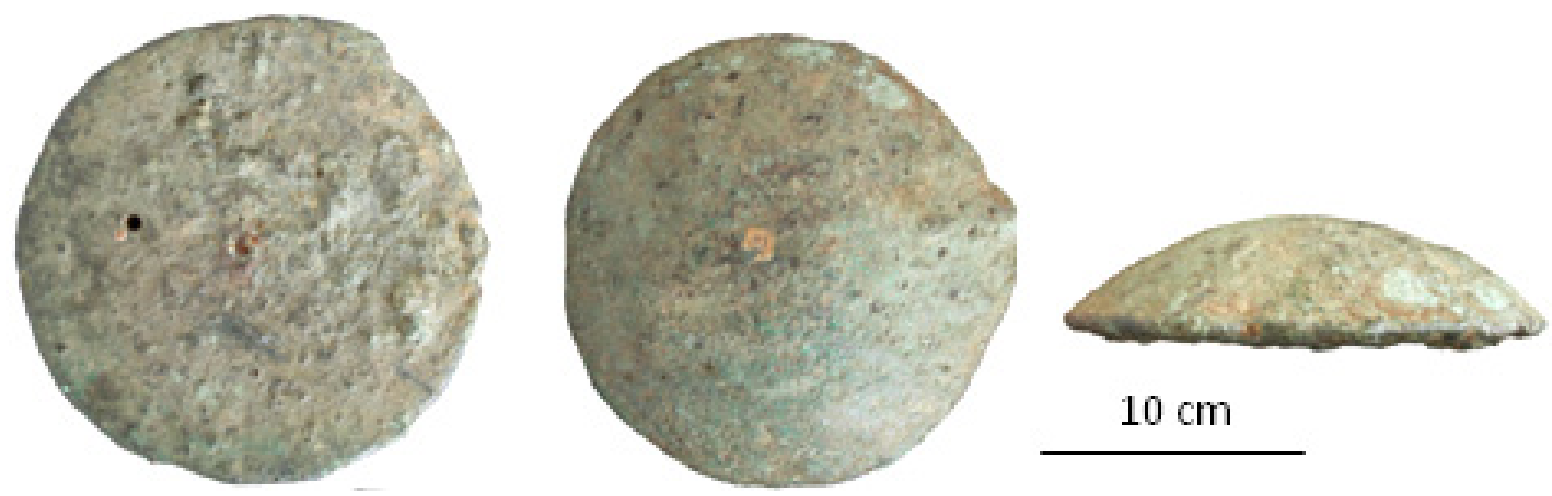

$10 \mathrm{~cm}$

Fig. 9 - Galette plano-convexe de bronze (dépôt de Menez Tosta, Gouesnac'h, Finistère).

Fig. 9 - Bronze plano-convex sample (Menez Tosta hoard, Gouesnac'h, Finistère). 


\section{QUELQUES RELECTURES DES DÉPÔTS}

$\mathrm{N}$ ous avons effectué quelques visites dans les musées afin de revoir les objets des dépôts à la lumière de cette nouvelle typologie. Ainsi, certains lingots de cuivre et déchets de fonderie peuvent avoir été complètement « oubliés ». C'est le cas pour le dépôt de Vern-sur-Seiche (Ille-et-Vilaine) pour lequel les inventaires précédents ne révèlent aucun lingot ni déchet de fonderie (Briard, 1991). Cependant, l'inventaire du musée les mentionne, mais avec des erreurs puisque certains sont décrits sous le terme de « scorie » alors qu'il s'agit de fragments de lingots plano-convexes. Les culots de fonte se révèlent être des masselottes et un fragment de lingot plano-convexe.

Pour d'autres dépôts, les résidus de fonderie sont vaguement mentionnés sans précision du nombre. Ainsi pour le dépôt de Kergadavarn à Plouguerneau, Finistère (Briard, 1991), l'examen a montré la présence de quatre lingots plano-convexes de cuivre, de quatre coulures sur un sol, d'une masselotte et de cinq objets indéterminés.

Certains inventaires, bien qu'assez anciens peuvent être assez fidèles à la réalité. C'est le cas du dépôt de Kerlouan (Finistère) pour lequel il a été listé dix fragments de lingots plano-convexes et des résidus de fonderie de bronze dont quatre fonds de creusets (Briard et al., 19641965). L'examen récent a permis de confirmer la présence des dix fragments de lingots plano-convexes de cuivre. Les résidus de fonderie se composent de quatre coulures sur un sol et neuf masselottes.

\section{DE L'IMPORTANCE D'IDENTIFIER LES LINGOTS DE CUIVRE}

$E^{n}$ n première approche, il est vrai que ces objets n'apportent aucune aide pour la détermination chronologique des dépôts. Est-ce autant une raison pour les négliger? Car d'autres pistes de recherche peuvent être suivies, apportant des renseignements différents mais tout aussi fondamentaux.

L'identification des lingots de cuivre et leur caractérisation morphologique sont un sujet de recherche particulièrement important. En effet, les lingots de cuivre ne proviennent pas de l'Ouest de la France où ils sont pourtant trouvés en grand nombre. Dans le Massif armoricain, la quantité de minerai de cuivre a toujours été insuffisante pour assurer la production imposante des objets, au moins à partir de l'époque du Bronze moyen. Les lingots sont donc importés d'autres régions productrices. Ces régions de production sont, au moins partiellement, connues pour cette époque, entre autres : Copa Hill (Timberlake, 1991) ou Great Orme (Dutton et al., 1994) au pays de Galles, peutêtre Mont Gabriel en Irlande (Brindley et Lanting, 1994), le district minier de Mauken dans le Tyrol autrichien (Schibler et al., 2011), mais actuellement il est toujours impossible de savoir réellement de quelles régions exactement provient le métal ayant servi à la fabrication de ces milliers d'objets.
En France, différentes formes de lingots ont déjà été identifiées. On pensera immédiatement aux lingots planoconvexes décrits dans cette étude, trouvés dans l'Ouest de la France, dont la taille est relativement standardisée avec un diamètre moyen aux environs de $17 \mathrm{~cm}$ (entre 14 et $21 \mathrm{~cm}$ ). Mais d'autres types de lingots se différencient par des caractères qui deviennent assez évidents une fois que l'on commence à les étudier dans le détail. Par exemple, le dépôt de Saint-Jean-aux-Bois, Oise, contient des exemplaires de lingots en forme de galettes circulaires aplaties larges de plus de $30 \mathrm{~cm}$ de diamètre ${ }^{(1)}$. Il fut un temps où ces lingots auraient tout simplement été qualifiés de planoconvexes. Cette simple description aurait alors produit une confusion, ces lingots auraient pu être rapprochés de ceux de l'Ouest de la France alors qu'il est évident qu'ils sont différents de ces derniers. On peut émettre l'hypothèse que des régions différentes du territoire français ont pu importer du cuivre, sous forme de lingots, de plusieurs régions productrices européennes. Ces dernières possèderaient alors leurs propres développements techniques conduisant à des formes différentes de lingots.

En comparant les lingots de cuivre trouvés sur le territoire français avec ceux trouvés dans des régions européennes productrices de cuivre, des ressemblances pourraient permettre d'émettre des hypothèses quant à leur origine. Une première étude rapide montre que la ressemblance des lingots plano-convexes étudiés ici avec des lingots provenant d'Angleterre est importante (Roberts et Veysey, 2011). Cependant, les lingots de cette époque retrouvés dans les îles Britanniques se concentrent essentiellement au Sud, au Sud-Est et à l'Est, ce qui les place en totale déconnexion avec les zones de production localisées en Irlande, en Cornouailles, au pays de Galles et en Écosse (Northover, rapport inédit). Il est alors difficile de confirmer l'origine. Ailleurs, les lingots retrouvés sur l'ensemble de la péninsule Ibérique présentent également de larges similitudes avec ceux présentés ici, même si des lingots présents dans quelques dépôts semblent être de taille moindre (Gomez Ramos, 1993; Montero Ruiz et al., 2011). Des disparités dans la typologie et la métrologie des lingots sont néanmoins parfaitement visibles, avec l'exemple des haches-lingots trouvées à l'est de la péninsule (Renzi, 2010). Les lingots planoconvexes semblent donc être des objets assez communs sur l'ensemble de l'Europe (Mordant, 1996) et seule une description macroscopique fine et homogénéisée de ces ensembles pourrait peut-être permettre de distinguer les productions de régions différentes.

Enfin, il est intéressant de noter que sur la façade atlantique, à l'âge du Fer, les dépôts ne contiennent plus guère que des haches à douille plus ou moins plombeuses. Ce sont les haches à douille de type armoricain qui ont été longtemps attribuées au Bronze final et dont on sait maintenant qu'elles ont été produites à l'âge du Fer (Gomez de Soto et al., 2009). La variété des objets dans ces dépôts cesse alors. La taille et la forme des lingots de cuivre ne sont plus connues. Par contre, des lingots plano-convexes de même type que ceux décrits dans ce travail se rencontrent dans le Sud de la France, en particulier dans le Languedoc occiden- 
tal (Mazière et Puig, 2002), mais aussi dans les îles Baléares et dans la vallée de l'Erbre (Gomez Ramos, 1993).

\section{CONCLUSION}

L es lingots de cuivre et les déchets de fonderie sont des objets omniprésents dans les dépôts métalliques terrestres de l'horizon de l'épée à pointe en langue de carpe du Bronze final IIIb, en particulier dans les régions proches de la façade atlantique. Et pourtant, ils ont été systématiquement sous-étudiés et même sous-référencés. La description de ces dépôts est, dans presque tous les cas, incomplète ou au mieux imprécise. L'étude précise de ces matériaux nous apporte pourtant de précieux renseignements sur les techniques métallurgiques employées par les bronziers de l'époque. Notamment il est ainsi possible d'approcher la forme de certains creusets à travers l'observation de leur moulage métallique. Une comparaison de ces formes à l'échelle nationale voire européenne pourrait peut être permettre de mettre en évidence des technologies de fonte différentes. Toujours dans le domaine de l'atelier de bronzier, la description précise des masselottes, et notamment des bavures de coulées peut permettre une certaine reconstitution des moules et appréhender ainsi la complexité de conception de certains d'entre eux.

Des données concernant l'organisation à grande échelle de la production et des voies d'échange pourraient également être obtenues en s'attachant à la description des lingots de cuivre. En effet, dans l'optique où chaque grande région production de cuivre peut mettre en place des techniques de réduction et de fonte particulières, il serait peut-être possible de visualiser ces échanges entre zones de production et zones de consommation à travers l'étude typologique fine des lingots de cuivre à l'échelle européenne, voire plus.

Remerciements : Nous remercions le conseil général du Finistère via la conservation départementale du Patrimoine et des Musées et le musée départemental Breton, pour avoir donné accès aux collections et notamment aux dépôts de Gouesnac'h. Nous remercions également José Gomez de Soto pour la relecture attentive qu'il a effectuée sur ce travail.

\section{NOTES}

1. Dépôt non publié, information du service régional de l'Archéologie de Picardie.

\section{RÉFÉRENCES BIBLIOGRAPHIQUES}

BLANCHET J.-C. (1984) - Les premiers métallurgistes en Picardie et dans le Nord de la France, Paris, Société préhistorique française (Mémoires, 17), $608 \mathrm{p}$.

Boulud S., FILY M. (2009) - Les dépôts métalliques de l'extrême fin du Bronze final en Bretagne : nouvelle évaluation des données à la lumière des découvertes récentes, in $\mathrm{A}$. Daubigney, P.-Y. Milcent, M. Talon, J. Vital (dir.), De l'âge du Bronze à l'âge du Fer en France et en Europe occidentale ( $X^{e}-V I I^{e}$ siècle av. J.-C.). La moyenne vallée du Rhône aux âges $d u$ Fer, actes du $\mathrm{XXX}^{\mathrm{e}}$ colloque international de l'AFEAF, co-organisé avec l'APRAB (Saint-Romain-enGal, mai 2006), Dijon, RAE (supplément à la Revue archéologique de l'Est, 27), p. 284-298.

Bourgeois J., Cherreté B. (2005) - L'âge du Bronze et le premier âge du Fer dans les Flandres occidentale et orientale (Belgique), in J. Bourgeois et M. Talon (éd.), L'âge du Bronze du Nord de la France dans son contexte européen, Paris, CTHS, p. 43-81.

Briard J., Bourhis J., Giot P.-R. (1964-1965) - Analyses spectrographiques d'objets préhistoriques et antique, $I^{\text {re }}$ série, Rennes, laboratoire d'Anthropologie préhistorique (Travaux), $123 \mathrm{p}$.

Briard J. (1966) - Le dépôt de la Prairie de Mauves à Nantes, Rennes, laboratoire d'Anthropologie préhistorique (Travaux, 59) p.

Briard J., (1971) - Un dépôt de l'âge du Bronze final : le Jardin des Plantes à Nantes (Loire-Atlantique), Nantes, direction des Antiquités préhistoriques (Études préhistoriques et protohistoriques Pays de la Loire, 2), 35 p.
BRIARD J. (1984) - L'outillage des fondeurs de l'âge du Bronze en Armorique, Paléométallurgie de la France atlantique, age du Bronze, Rennes, laboratoire « Anthropologie, Préhistoire, Protohistoire, Quaternaire armoricains » (Travaux), p. 139-168.

BriARD J. (1991) - Le groupe de l'épée à pointe en langue de carpe en Armorique : une révision » in $\mathrm{C}$. Chevillot et A. Coffyn (dir.), L'âge du Bronze atlantique, ses faciès de l'Écosse à l'Andalousie et leur relation avec le Bronze continental et la Méditerranée, actes du colloque (Beynacet-Cazenac, 1990), Beynac-et-Cazenac, Association des musées du Sarladais, p. 125-144.

Brindley A., Lanting J. (1994) - The Radiocarbon Chronology of Mines 3 and 4, Mount Gabriel, in W. O'Brien (éd.), Mount Gabriel. Bronze Age Mining in Ireland, Galway, Galway University Press, p. 281-288.

Chatelier P. du (1899) - Le Bronze dans le Finistère, Bulletin de la Société archéologique du Finistère, 26, p. 250-303.

Closmadeuc G. de (1863) - Notes et considérations archéologiques sur les bronzes gaulois découverts aux environs de Questembert, Bulletin de la Société polymathique du Morbihan, p. 10-30.

Coffyn A. (1971) - Le Bronze final et le début du premier âge du Fer autour de l'estuaire girondin, thèse de doctorat, université de Bordeaux, 5 vol.

Coffyn A., Gomez de Soto J., Mohen J.-P. (1981) - L'apogée du Bronze atlantique, le dépôt de Vénat, Paris, Picard, $238 \mathrm{p}$. 
Cordier G. (1996) - Le dépôt de l'âge du Bronze final du «Petit-Villatte » à Neuvy-sur-Barangeon (Cher) et son contexte régional, Joué-lès-Tours, chez l'auteur, $99 \mathrm{p}$.

Dutton L. A., Fasham P. J., Jenskins D. A., Caseldine A. E., Hamilton-Dyer S. (1994) - Prehistoric Copper Mining of the Geat Orme, Llandudno, Gwynedd, Proceedings of the Prehistoric Society, 60, p. 245-286.

FisCHER V. (2012) - Les bronzes en contexte palafittique sur les rives du Léman et des Trois Lacs (Suisse occidentale), Lausanne, CAR (Cahiers d'archéologie romande, 128), 175p.

Fily, M. (2009) - Les dépôts de la fin de l'âge du Bronze final de Kergaradec à Gouesnac'h (Finistère) : note préliminaire sur une découverte récente de l'horizon métallique de l'épée à pointe en langue de carpe, Bulletin de la Société préhistorique française, 106, 1, p. 95-107.

GAuthier É. (2003) - Étude de la variabilité de la composition des dépôts de bronze, en France orientale, aux X ${ }^{\mathrm{e}}$ et IX ${ }^{\mathrm{e}}$ siècles avant notre ère, Revue archéologie de l'Est, 52, p. $19-44$.

Gauthier É. (2009) - La consommation et la circulation du métal à l'âge du Bronze dans le Jura et les plaines de la Saône, in A. Richard, P. Barral, A. Daubigney, G. Kaenel, C. Mordant et J.-F. Piningre (dir.), L'isthme européen RhinSaône-Rhône dans la Protohistoire. Approches nouvelles en hommage à Jacques-Pierre Millotte, actes du colloque en hommage à J.-P. Millotte (Besançon, 16 -18 octobre 2006), Paris, Les Belles Lettres (Annales littéraires de l'université de Besançon, série Environnement, sociétés et archéologie), p. $145-159$.

Guilaine J. (1972) - L'âge du Bronze en Languedoc occidental, Roussillon, Ariège, Paris, Klincksiek (Mémoires de la Société préhistorique française, 9), $460 \mathrm{p}$.

Gomez de Soto J. (1995) - Le Bronze moyen en Occident : la culture des Duffaits et la civilisation des tumulus, Paris, Picard (L'âge du Bronze en France, 5), 375 p.

Gomez de Soto J., Milcent P.-Y. (2000) - De la Méditerranée à l'Atlantique : échanges et affinités culturelles entre le nord-ouest (Armorique, Centre-Ouest, Limousin) et le SudOuest de la France (principalement Languedoc occidental) de la fin $d u X^{e}$ au Ve s. avant J.-C., Lattes, ARALO (Monographies d'archéologie méditerranéenne, 7), p. 351-371.

Gomez De Soto J., Bourhis J.-R., Marcigny C., Menez Y., Rivallain J., Verron G. (2009) - Pour en finir avec le Bronze final? Les haches à douille de type armoricain en France, in A. Daubigney, P.-Y. Milcent, M. Talon et J. Vital (dir.), De l'âge du Bronze à l'âge du Fer en France et en Europe occidentale ( $X^{e}$-VII ${ }^{e}$ siècle av. J.-C.), actes du XXX ${ }^{e}$ colloque de l'AFEAF, co-organisé avec l'APRAB (SaintRomain-en-Gal, 2006), Dijon, RAE (supplément à la Revue archéologique de l'Est, 27), p. 507-512.

Gomez Ramos P. (1993) - Tipología de lingotes de metal y su hallazgo en los depósitos del Bronce final de la Península Ibérica, Madrid, Universidad Autónoma (Cuadernos de Prehistoria y Arqueología, 20), p. 73-105.

Lagarde, D. (1863) - Armes et ustensiles celtiques découvert en 1861 dans la commune de Lampaul-Plouarzel (Finistère), Bulletin de la Société académique de Brest, 3, p. 125-130.
Le Men R.-F. (1876) - Statistiques monumentales du Finistère : époque celtique, Bulletin de la Société archéologique du Finistère, 4, p. 85-136.

Marcigny C., Guesquière E., Clement-Sauleau S., VerNEY A. (2005) - L'âge du Bronze en Basse-Normandie : définition par le mobilier céramique, une première tentative, in J. Bourgeois et M. Talon (dir.), Actes du $125^{e}$ congrès national des sociétés historiques et scientifiques (Lille, 2000), Paris, CTHS, p. 303-332.

Marsille L. (1911) - Le Bronze dans l'arrondissement de Quimperlé, Bulletin de la Société polymathique du Morbihan, p. 77-91.

Marsille L. (1913) - Liste des dépôts de l'âge du Bronze dans le Morbihan, Bulletin de la Société polymathique du Morbihan, p. 49-109.

Marsille L. (1921) - Les dépôts de l'âge du Bronze dans le Morbihan, Bulletin de la Société polymathique du Morbihan, extrait, p. 1-27.

Maziere F., Puig C. (2002) - Un dépôt de bronze du premier âge du Fer en Roussillon : Les Teixons (Pollestres, Pyrénées Orientales), Cypsela, 14, p. 229-236.

Mercurin R. (2011) - Les dépôts d'objets en bronze dans les Alpes-Maritimes à l'âge du Bronze final : état des lieux et perspectives de recherche, in D. Garcia (éd.), L'âge du Bronze en Méditerranée. Recherches récentes, Paris, Errance, p. 131-154.

Milcent P.-Y. (1998) - Le petit Villatte à Neuvy-sur-Barangeon (Cher) : lecture d'un dépôt complexe, in C. Mordant, M. Pernot et V. Rychner (dir.), L'atelier du bronzier en Europe du XX'e s. au VIII' s. avant notre ère, III. Production, circulation et consommation du bronze, actes du colloque « Bronz'96 » (Neuchâtel et Dijon, 1996), Paris, CTHS et Dijon, université de Bourgogne, p. 55-69.

Milcent P.-Y. (2012) - Le temps des élites en Gaule atlantique : chronologie des mobiliers et rythmes de constitution des dépôts métalliques dans le contexte européen (XIII ${ }^{e}-V^{2}{ }^{e} s$. av. J.-C.), Rennes, PUR (Archéologie et culture), 253 p.

Mohen J.-P. (1977) - L'âge du Bronze dans la région de Paris : catalogue synthétique des collections conservées aux musée des Antiquités nationales, Paris, Éditions des musées nationaux, $263 \mathrm{p}$.

Mordant C. (1996) - Dépôts de bronzes et territoires à l'âge $\mathrm{du}$ Bronze en Bourgogne (XVII-IX ${ }^{\mathrm{e}}$ siècle avant J.-C.), in C. Mordant, M. Pernot et V. Rychner (dir.), L'atelier du bronzier en Europe du XX ${ }^{e}$ s. au VIII ${ }^{e}$ s. avant notre ère, III. Production, circulation et consommation du bronze, actes du colloque « Bronz'96 » (Neuchâtel et Dijon, 1996), Paris, CTHS et Dijon, université de Bourgogne, p. 185-210.

Mordant C. (2001) - Le dépôt d'Arcy-sur-Cure et les dépôts du Bronze final IIIb de la Bourgogne occidentale, in $D u$ monde des chasseurs à celui des métallurgistes, Rennes, ADRAOF (Supplément à la Revue archéologique de l'Ouest), p. 207-216.

Montero-Ruiz I., Rovira Hortala M. C., Renzi M., Murillo-Barroso M., Hunt M., Gener M., Castanye P. (2011) - Lingotes plano-convexos de cobre en la primera mitad del I milenio ac en la Península Ibérica, Boletin de Asociacion espanola de amigos de la Arqueologia, 46, p. 99-119. 
Mortillet G. de (1894) - Cachettes de l'âge du Bronze en France, Bulletin de la Société française d'anthropologie de Paris, 5, p. 298-340.

Needham S. (1980) - An Assemblage of Late Bronze Age Metalworking Debris from Daiton, Devon, Proceedings of the Prehistoric Society, 46, p. 177-215.

Northover J. P. (rapport non publié) - Metallurgical analysis of ingots hoards for Essex.

Parenteau F. (1868) - Découverte du Jardin des Plantes de Nantes, Bulletin de la Société archéologique de Nantes, 8, p. 19-46.

Pautreau J.-P., 1979 - Le Chalcolithique et l'âge du Bronze en Poitou (Vendée, Deux-Sèvres, Vienne), Poitiers, Centre d'archéologie et d'ethnologie poitevine, 2 vol., 434 p.

PAutreau J.-P. (1984) - Quelques aspects de la métallurgie en Poitou aux âges du Cuivre et du Bronze, in Paléométallurgie de la France atlantique, 1. Age du Bronze, Rennes, université Rennes 1 (Travaux du laboratoire « Anthropologie, Préhistoire, Protohistoire, Quaternaire armoricains »), p. 99-134.

Queixalos I., Menu M., Mohen J.-P. (1987) - Creusets pour la fonte des alliages à base cuivre du Bronze final au Fort Harrouard à Sorel Moussel (Eure-et-Loir), Bulletin de la Société préhistorique française, 84, p. 23-30.

RenZI M. (2010) - La producción de «lingotes-hacha» en el Levante peninsular: nueva valoración a partir de los materiales de La Fonteta (Guardamar del Segura, Alicante), Revista de Arqueologia de Ponent. 20, p. 127-143.

Roberts B., Veysey C. (2011) - Benjamin Roberts and Charlotte Veysey Reveal the Significance of a Bronze Age Shipwreck Found Off the Coast of Devon, British Museum Magazine, p. 44-45.

Schibler J. , Breitenlechner E., Deschler-Erb S. , Goldenberg G., Hanke K., Hiebel G., Plogmann H. H., Nicoluss K., Marti-Gradel E., Pichler S., Schmidt A., Stefan Schwarz S., Stopp B., Oeggl K. (2011) - Miners and Mining in the Late Bronze Age: a Multidisciplinary Study from Austria, Antiquity, 85, p. 1259-1278.

Timberlake S. (1991) - New Evidence for Early Prehistoric Mining in Wales, in P. Budd, B. Chapman, C. Jackson,
R. Janaway et B. S. Ottaway (éd.), Proceedings of a Conference on the Application of Scientific Techniques to Archaeology (Bradford 1989), Oxford, Oxbow (Oxbow Monograph 9), p. 179-193.

Veber C. ( 1996) - Introduction à l'étude du dépôt de Farébersviller (Moselle) et production métallique du Bronze final IIIb en Lorraine, in C. Mordant, M. Pernot et V. Rychner (dir.), L'atelier du bronzier en Europe du XX e s. au VIII' $s$. avant notre ère, III. Production, circulation et consommation du bronze, actes du colloque « Bronz'96 » (Neuchâtel et Dijon, 1996), Paris, CTHS et Dijon, université de Bourgogne, p. 41-54.

Verney A. (1990) - Le dépôt de Challans, Vendée, Bulletin de la Société préhistorique française, 87, 10-12, p. 396-417.

Villiers du Terrage, É. de (1896) - Note sur une cachette de fondeur de l'âge du Cuivre découverte à Tourc'h, Bulletin de la Société archéologique du Finistère, 23, p. 103-109.

Cécile Le Carlier de Veslud UMR 6566 « CReAAH»

laboratoire «Archéosciences » université Rennes 1

Campus de Beaulieu, 35042 Rennes cedex cecile.lecarlier@univ-rennes1.fr

Laura Edme UMR 6566 « CReAAH» laboratoire «Archéosciences » université Rennes 1,

Campus de Beaulieu, 35042 Rennes cedex laura.edme@laposte.net

Muriel FiLY centre départemental de l'Archéologie, conseil général du Finistère 16 route de Térénez, 29590 Le Faou muriel.fily@cg29.fr 